\title{
Role of Autophagy-Related Gene atg22 in Developmental Process and Virulence of Fusarium oxysporum
}

\author{
A. Rehman Khalid ${ }^{1,2, *}$, Shumin Zhang ${ }^{1}$, Xiumei Luo ${ }^{1}$, Khalid Mehmood ${ }^{3}$, Junaid Rahim ${ }^{3}$, \\ Hamayun Shaheen ${ }^{4}$, Pan Dong ${ }^{1}$, Dan Qiu ${ }^{1, *}$ and Maozhi Ren ${ }^{1, *}$ \\ 1 School of Life Sciences, Chongqing University, Chongqing 401331, China; youran@cqu.edu.cn (S.Z.); \\ aoamateen319@yahoo.com (X.L.); haseeb78692@yahoo.com (P.D.) \\ 2 Department of Plant Pathology, University of Poonch AJK, Rawalkot 12350, Pakistan \\ 3 Department of Entomology, University of Poonch AJK, Rawalkot 12350, Pakistan; \\ kmmaldial@yahoo.com (K.M.); junaidrahim47@yahoo.com (J.R.) \\ 4 Department of Botany, The University of Azad Jammu \& Kashmir, Muzafarabad13100, Pakistan; \\ hamayun@hotmail.com \\ * Correspondence: arkhalid@cqu.edu.cn (A.R.K.); quidan@outlook.com (D.Q.); renmaozhi@cqu.edu.cn (M.R.)
}

Received: 30 March 2019; Accepted: 6 May 2019; Published: 13 May 2019

\begin{abstract}
Autophagy is a universal catabolic process preserved in eukaryotes from yeast to plants and mammals. The main purpose of autophagy is to degrade cytoplasmic materials within the lysosome/vacuole lumen and generate an internal nutrient pool that is recycled back to the cytosol during nutrient stress. Here, Fusarium oxysporum was utilized as a model organism, and we found that autophagy assumes an imperative job in affecting the morphology, development, improvement and pathogenicity of F. oxysporum. The search of autophagy pathway components from the F. oxysporum genome database recognized putative orthologs of 16 core autophagy-related (ATG) genes of yeast, which additionally incorporate the ubiquitin-like protein atg22. Present study elucidates the unreported role of Foatg22 in formation of autophagosomes. The deletion mutant of Foatg22 did not demonstrate positive monodansylcadaverine (MDC) staining, which exposed that Foatg22 is required for autophagy in F. oxysporum. Moreover, the $\Delta$ Foatg22 strains exhibited a decrease in hyphal development and conidiation, and reduction in pathogenicity on potato tubers and leaves of potato plant. The hyphae of $\Delta$ Foatg 22 mutants were less dense when contrasted with wild-type (WT) and overexpression (OE) mutants. Our perceptions demonstrated that Foatg22 might be a key regulator for the control of dry rot disease in tuber and root crops during postharvest stage.
\end{abstract}

Keywords: Fusarium oxysporum; autophagy; potato dry rot; filamentous fungi; virulence

\section{Introduction}

The fungus Fusarium oxysporum is the most common pathogen infection of tubers and roots [1], which causes Fusarium wilting at the planting stage and dry rot at the storage stage, impacting its nutritive and economic value [2,3]. Also, this fungus can cause severe reductions in crop yield, often estimated between $6 \%$ and $25 \%$ annually throughout the world. Significant yield losses of $60 \%$ are recorded worldwide in storage conditions [4-6]. Fusarium oxysporum can be seed-borne or soil-borne; it can behave as a vascular colonization pathogen, and act as a decomposer [7,8]. Dry rot disease can be identified by shrinking, shriveling, and lesions on the tuber/root, with brown to black characteristics in the internal part of tuber/root $[7,9,10]$. The pathogen penetrates the tissues such as roots, tubers, and leaves through wounds and then induces rot infection. The infected tissues become dark red by forming streaks which rise to ground level. With the passage of time, the old leaves become yellow or 
flaky and then detach from the plant [11]. It was demonstrated that, in F. oxysporum, during vegetative growth, autophagy controls the number of nuclei in the hyphal compartment and is also essential for virulence [12].

Autophagy is a coordinated procedure which is liable for the quick declension of vast parts of the cytoplasm in the vacuolar lumen $[13,14]$. On the other hand, the recycling of degradation products, such as amino acids, generated in autophagy toward the cytoplasm is critical to cell physiology; however, the molecular details of this process remain to be adequately determined. The fission yeast Schizosaccharomyces pombe contains a homolog of Saccharomyces cerevisiaeatg22p which is a vacuolar membrane protein involved in the breakdown of autophagic bodies during autophagy [15]. The deduced amino-acid sequence of $S$. pombeatg 22 shared $21 \%$ similarity and $42 \%$ identity with S. cerevisiaeatg 22 . SOSUI investigation (http://bp.nuap.nagoya-u.ac.jp/sosui/) proposed that S. pombeATG22 has eleven transmembrane helices. In spite of the fact that S. pombeatg22 is essential for germination of spores [13], the criticalness of $a t g 22$ in the cell compartmentalization of amino acids remains to be explored. Autophagy plays a fundamental role in cell differentiation and the development of pathogenicity in phytopathogenic fungi [16-20]. In rice blast fungus, Magnaporthe oryzae, the analysis of autophagy-related genes demonstrated that mutants lacking any of these genes failed to cause disease. Loss of any of the atg genes, except Fgatg17, prevented the fungus from causing Fusarium head blight disease in wheat [21]. The autophagy affects the formation of appressorium, which directly contributes to the development of diseases caused by fungus Magnaporthe oryzae [22-24]. Autophagy also affects the virulence of Ustilago maydis, the cause of corn smut, and penetrates the host through appressorium [20]. Studies reported that different autophagy-related genes were involved in the pathogenicity of Verticillium dahlia, including $V d P K A C 1, V G B, V M K 1, V G B, V d S N F 1$, and VdPKAC1 [25-28]. Here, we explored the function of autophagy-related gene atg22 in the development and pathogenicity of F. oxysporum. We generated gene deletion mutant $\Delta a t g 22$ by adopting a target gene replacement technique and generated an overexpressed mutant of $a t g 22$. The role of these mutants in the development of aerial hyphae, conidial formation, and pathogenicity was evaluated.

\section{Materials and Methods}

\subsection{Isolation of Fungus and Culture Conditions}

The strains of Fusarium oxysporum f. sp. lycopersici were isolated from diseased tubers of potato, and their identity was confirmed by plant infection assay before being used as wild-type (WT) strains in the present study. These strains were sensitive to HygB when used at a concentration greater than $30 \mathrm{mg} / \mathrm{mL}$. A conidial suspension of strains was prepared and then stored at $-80{ }^{\circ} \mathrm{C}$ with the addition of $50 \%$ glycerol. For further use, the conidia were grown again on fresh potato dextrose agar(PDA)medium at $25{ }^{\circ} \mathrm{C}$ For the preparation of mycelium and conidia, PDA liquid medium was used. The A. tumefaciens strains of agrobacteria GV3101 were used for the transformation of conidia of F. oxysporum. The strains of agro bacterium $A$. tumefaciens were grown on LB media.

\subsection{Generation of Deletion Mutants}

The Foatg22 deletion mutant was generated with fusion PCR [29]. The Foatg22 deletion mutant was created using HygB resistance cassette with the replacement of the open reading frame (ORF). The first 1000-bp upstream fragments, the HygB resistance cassette of $1040 \mathrm{bp}$ from psilent- 1 vector, the HygB resistance cassette from psilent-1 vector, and the downstream fragment of 1000-bp Foatg22 were amplified with three pairs of primers, i.e., P1/P2, P3/P4, and P5/P6, respectively. After that, three fragments were fused with upstream-Hph-downstream, and then restriction enzyme digestion was performed with enzymes AsiSI and Sbflby ligation with vector PPk2 (Figure S1A, Supplementary Materials). The final recombinant plasmid of PPk2-U-Hph-D was transferred into WT according to a previously reported method [30]. The PDA media which was supplemented with $50 \mu \mathrm{M}$ F2du (5-fluoro-2'-deoxyuridine) and $50 \mathrm{mg} / \mathrm{mL}$ HygB was used for screening of transformants. 
The transformants were confirmed through PCR screening with F-hph/R-hph. Then, qRT-PCR was used for identification of the deletion mutant followed by PCR screening. For identification of whether HygB was a single copy of the inserted mutant, we used F- $h p h / \mathrm{R}-h p h$ to $1021 \mathrm{bp}$.

\subsection{Overexpression of Foatg22 Mutant Strains}

The complementary DNA (cDNA) of F. oxysporum encompassed the complete Foatg22 ORF and it was amplified with total RNA using primers atg22F/R, which corresponded to seven initial codons of ORF, restriction site NotI, and additional cytosine. For reverse complementation, atg22-12 corresponded to the last seven codons of the restriction site $S b f \mathrm{I}$, as well as additional cytosine. Then, the resulting band was cloned into the vector $\mathrm{p} 8 \mathrm{GWN}$. This plasmid was used as a template for amplification of PCR. The primer atg22-F corresponded to the first eight codons with $S b f \mathrm{I}$ and AscI restriction sites (Table S1, Supplementary Materials). The amplified fragment of DNA was cloned into the F303 vector, resulting in plasmid Foatg22/F303. A 2.8-kb fragment of Foatg22 fusion under the control of the trpC promoter and the $\operatorname{trpC}$ terminator was obtained by PCR amplification using primer pair M13F and M13R, as mentioned in Table S1 (Supplementary Materials), and it was later used for protoplast strain formation (Figure S1B, Supplementary Materials).

\subsection{Fungal Transformation}

According to a previously described method, the agro bacterium tumefaciens mediated transformation (ATMT) process was performed with some modifications [30]. The strains of A.tumefaciens GV3101 containing PPk2 vector were grown on media amended with PDA at $28^{\circ} \mathrm{C}$ and allowed to grow. When the optical density of strains at $660 \mathrm{~nm}\left(\mathrm{OD}_{660}\right)$ reached 0.5 , the culture was mixed in conidial suspension $\left(10^{7}\right.$ conidia/mL) with equal concentration and then diluted to $\mathrm{OD}_{600}=0.15$ in the induction medium (IM) which contained $200 \mathrm{mM}$ acetosyringone (AS), before being cultured on an orbital shaker at $200 \mathrm{rpm}$ for $6 \mathrm{~h}$ at $28^{\circ} \mathrm{C}$. Then, $250 \mathrm{mM}$ of the mixture was kept on nitrocellulose filters (diameter, $80 \mathrm{~mm}$, pore size, $0.45 \mathrm{~mm}$; Whatman, Tokyo, Japan) for $48 \mathrm{~h}$ on co-cultivation medium. These filters were further transferred to a selective medium which was amended with cefotoxime ( $500 \mathrm{mg} / \mathrm{mL})$ and HygB $(50 \mathrm{mg} / \mathrm{mL})$ to defeat cells of $A$. tumefaciens. The transformants were selected randomly after seven days and then transferred on PDA medium which was enriched with HygB ( $50 \mathrm{mg} / \mathrm{mL}$ ).

\subsection{Evaluation of Radial Growth, Conidiation, Formation, and Germination}

In the present study, to evaluate conidial formation and radial growth of strains, the PDA medium was used which contained $50 \mathrm{mg} / \mathrm{mL}$ HygB. From a 10-day-old culture, the conidia of fungus was harvested and then filtered from lens paper containing two layers, before being re suspended with the concentration of $1 \times 10^{7}$ spores $/ \mathrm{mL}$ in sterile water. The $5-\mu \mathrm{L}$ conidial suspension of WT, Foatg $22 \Delta$, and overexpression strains was inoculated in each flask and plate. The plates were incubated at $25^{\circ} \mathrm{C}$. The diameter of the colony and the color of strains were observed and measured every day. To observe the conidial germination, in $1 \mathrm{~mL}$ of potato dextrose agar (PDB) medium, $10^{2}$ conidia were inoculated with continuous shaking at $150 \mathrm{rpm}$, and conidial germination was observed at 7, 12, 21, 28, 36, and $48 \mathrm{~h}$. At $12 \mathrm{~h}$, using a blood counting chamber, the germination rate of conidia was calculated [31]. To analyze the data, the software SPSS 15.0 for Windows ${ }^{\mathrm{R}}$ (LEAD Technologies, Inc., Charlotte, NC, USA) was used. For statistical analysis, the Duncan post hoc test was used to observe the differences among strains atp $\leq 0.05$. For calcofluor white staining, $5 \times 10^{7}$ freshly obtain micro conidia were grown at $28{ }^{\circ} \mathrm{C}$ for $14 \mathrm{~h}$ in $5 \mathrm{~mL}$ of PDA with shaking at $170 \mathrm{rpm}$, and incubated in the dark for five minutes with $10 \mu \mathrm{m}$ of calcofluor white (CFW).

\subsection{Analysis of Autophagy}

In strains of $F$. oxysporum, to visualize the autophagy, $2.5 \times 10^{8}$ micro conidia of different strains were inoculated on PDA medium and allowed to grow at $28^{\circ} \mathrm{C}$ for $15 \mathrm{~h}$. The strains were washed with sterile water and then transferred into synthetic medium (SM) medium lacking a nitrogen source in the 
presence or absence of $4 \mathrm{mM}$ phenylmethylsulfonyl fluoride (PMSF) (Sigma, P7626). After starvation for $1 \mathrm{~h}$, the mycelium of strains was stained with monodansylcadaverine (MDC) (Sigma, D4008), at a concentration of $50 \mathrm{mM}$ in the dark for $30 \mathrm{~min}$. Then, strains were washed with water and observed under differential interference contrast (DIC) and epifluorescence microscopy.

\subsection{Analysis of Gene Expression}

The RNA of OEFoatg22 and $\triangle$ Foatg22 mutants and WT of F. oxysporum was extracted to perform qRT-PCR. The total RNA was extracted using Magen (Hi Pure RNA mini Kit) according to the manufacturer's described protocol. The mixture of primer Script Rt Enzyme (Takara) and oligo (dT) primers was used with RNA. The cDNA was diluted to $100 \mathrm{ng} / \mathrm{L}$. The RT-PCR was run with 30 cycles on a Bio-Rad PTC0200 Peltier Thermal Cycler (Bio-Rad, Hercules, CA, USA). For an internal control, the EF1 $\alpha$ gene was amplified, and grayscale was used to analyze expression values. The qRT-PCR was performed and, as a template, first-strand cDNA was used with TB SYBR Supermix (Takara). As an endogenous control, EF1 $\alpha$ was used, and all reactions were carried out three times. To perform qRT-PCR, the following PCR protocol was adopted: initial denaturation at $95^{\circ} \mathrm{C}$ for $2 \mathrm{~min}$, followed by 40 cycles of $95^{\circ} \mathrm{C}$ for $10 \mathrm{~s}$, and annealing temperature for $30 \mathrm{~s}$. The annealing temperature for FOXG_04522 was $63.5^{\circ} \mathrm{C}$. To analyze data, the software CFX Manager ${ }^{\mathrm{TM}}$ was used. Normalized expression level was determined among mutants and WT using the comparative $\mathrm{Ct}$ method $\left(2^{-\Delta \Delta \mathrm{Ct}}\right)$, in which $\Delta \Delta \mathrm{Ct}=\left(\mathrm{Ct}_{\text {gene }}-\mathrm{Ct}_{18 \mathrm{srRNA}}\right)$ mutant $-\left(\mathrm{Ct}_{\text {gene }}-\mathrm{Ct}_{18 \mathrm{srRNA}}\right) \mathrm{WT}$. For RT-PCR, specific pairs of gene primers (RT-Foatg22F and RT-Foatg22R) were designed, as listed in Table S1 (Supplementary Materials). The experiments were performed three times with biological replicates.

\subsection{Pathogenicity Test}

For the tuber inoculation assay, healthy and uniform (100-120 g) potato tubers were used in the present study. Excessive soil and contamination was removed by washing the tubers. For surface sterilization, tubers were dipped for $10 \mathrm{~min}$ in a solution of sodium hypochlorite $(0.5 \%)$ and rinsed with sterile distilled water with three changes, before being cut into small slices and air-dried. The strains of WT (F. oxysporum\#1), Foatg22 , and Foatg22 overexpression mutant were incubated on potato dextrose agar (PDA) medium plates for 14 days in darkness. From each strain, conidia were collected and washed with pea broth. A $20-\mu \mathrm{L}$ drop of conidial suspension of $1 \times 10^{4}$ conidia/mL for WT, Foatg22 $\Delta$, and OEFoatg22 was inoculated on potato slices. Five leaves and slices of each potato were placed on moist filter paper in a dish, with three replicates used for each treatment. The inoculated potato slices and leaves were incubated at $27^{\circ} \mathrm{C}$ in dark/light conditions for five days. The sizes of each strain on potato slices were measured and analyzed with the Duncan post hoc test. Each experiment was performed twice.

\subsection{Optical and Epifluorescence Microscopy}

To perform epifluorescence and optical microscopic analysis, the aliquots of cells were embedded in 1\% agarose blocks and observed under the microscope (M2 Dual Cam) with an appropriate set of filters. The epifluorescence examination was performed using ultraviolet (UV) light (340 to $380 \mathrm{~nm}$ ) and the following filter blocks: MDC staining (G 365, FT 395, LP 420). To capture the images, a digital camera (EM512 Evolve Photometric) was used with Axiovision 4.8 software. Images were processed using Adobe Photoshop CS3.

\section{Results}

\subsection{Deletion and Overexpression Mutants of Foatg22 in F. oxysporum}

To study the function of Foatg22, target gene replacement was performed in the strains of F. oxysporum $\mathrm{f}$. sp. lycopersici. The hygromycin-resistant (HygR) transformants were analyzed by polymerase chain reaction (PCR) amplification with a specific pair of primers (Foatg22F and Foatg22R) 
on insertion flanking regions. The transformants showed an expected shift of the band corresponding to Foatg22 (Figure S2, Supplementary Materials). These deletion mutants were further confirmed by qRT-PCR with a specific primer pair (RT Foatg22F and RT Foatg22R) (Figure 1; Table S1, Supplementary Materials). Overexpression of Foatg22 was achieved by co-transformation with Foatg22 genes and different vectors, as confirmed by PCR analysis using specific pairs of primers (PtrpCF and Foatg22R) (Table S1, Supplementary Materials). The transformants showed the expected shift band corresponding to Foatg22 in overexpression (OE) mutants (Figure S2, Supplementary Materials). The transformants were further confirmed by qRT-PCR using a specific pair of primers (RT-Foatg22F and RT-Foatg22R) (Figure 1; Table S1, Supplementary Materials).

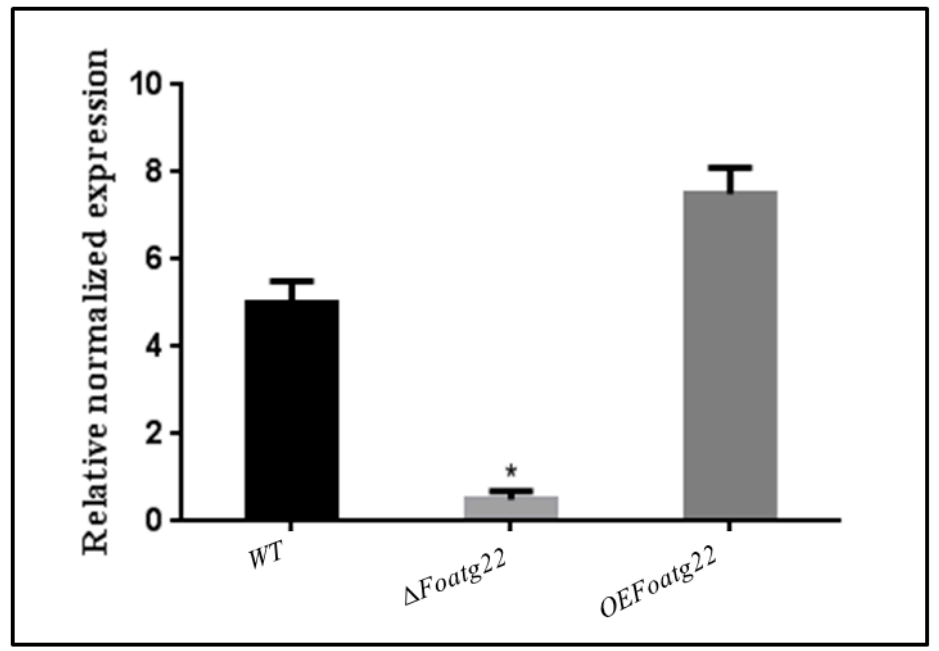

Figure 1. Quantitative real-time reverse-transcription polymerase chain reaction (qRT-PCR) analysis of wild-type (WT), $\Delta$ Foatg22, and overexpression strains. Three biological replicates were used for this study. The error bars show standard deviation. To perform statistical analysis, the Duncan post hoc test was used $(p<0.05)$.

\subsection{Role of ATG22 in Hyphal Formation}

The role of autophagy in several developmental stages of F. oxysporum was investigated through observations of autophagosomes. Three-day-old hyphae were collected for staining with monodansylcadaverine (MDC), a dye which is widely used for the detection of the autophagosome in cells [32,33]. In three-day-old hyphae, fluorescence was absent in the deletion mutant compared to wild type (WT), while fluorescence dots of the MDC staining remarkably increased in overexpression mutants (Figure 2). Moreover, we observed that MDC-stained fluorescence dots were similar to overexpression in the wild type (Figure 2). This process of formation of autophagosomes showed that, during the stages of hyphal formation in the wild-type strain and overexpression mutant, the autophagy process was activated. Furthermore, we investigated the role of ATG22 in phenotype changes in hyphae; the overexpression transformants and deletion mutants were examined for phenotype changes. The hyphal formation in the deletion mutant was significantly different from that in the wild-type and overexpression strains. The wild-type and overexpression strains exhibited dense hyphal formation compared to the deletion mutant (Figure S3A, Supplementary Materials). Moreover, after three days of inoculation, hyphal size was not significantly reduced in the deletion mutant compared to the overexpression and wild-type strains (Figure S3B, Supplementary Materials). Taken together, the present findings suggest that autophagy contributes to hyphal formation in F. oxysporum. 


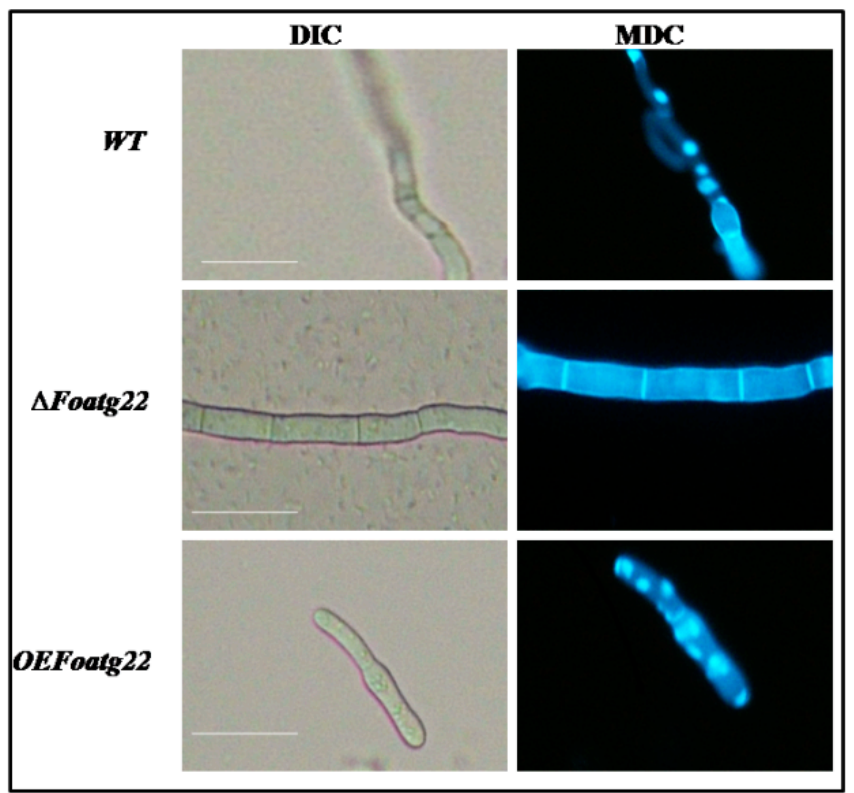

Figure 2. Autophagy formation in three-day-old hyphae of strains which were stained with monodansylcadaverine (MDC). Fluorescence dots show the formation of autophagosomes. Bars $=15 \mu \mathrm{m}$.

\subsection{Autophagy Contributes toMycelial Growth and Conidiation}

In several fungi, imperfect autophagy severely affects colony growth and conidial formation [33-37]. However, to investigate the role of autophagy in the growth and developmental process of F. oxysporumon different media, we adopted overexpression and knockout approaches. Firstly, we evaluated mycelial growth on potato dextrose agar (PDA). The mycelium of F. oxysporum mutants $\triangle F o a t g 22$ and OEFoatg 22 were inoculated in the center of plate and then allowed for grow, while the growth of mycelium was observed over a specific period of time. After two weeks of inoculation, the mycelium of the overexpression (OE) mutant covered approximately the whole surface of the plate, while in the deletion mutant, radial growth was suppressed slightly compared to the wild type (Figure 4A). However, in the deletion mutant, mycelial growth was significantly suppressed and colony diameter was reduced compare to wild type (WT) and the overexpression (OE) mutant (Figure 3B). This finding suggests that autophagy contributes to the mycelial growth of fungus F. oxysporum. Moreover, those strains which were grown under nutrient-limited conditions (SM diluted 1:1000) exhibited a faint mycelium with an increase in diameter, but aerial hyphae were not detectable, probably due to severe nutrient-limited conditions (Figure S4A, Supplementary Materials). However, the mycelial growth was significantly reduced in deletion mutants compared to wild-type and overexpression strains (Figure S4B, Supplementary Materials).

Furthermore, we determined conidial formation in both rich and minimal media. In rich nutrient media (both solid and liquid), after 14 days in solid and after two days in liquid media, the recovered micro conidia were significantly reduced in the deletion mutant compared to the wild type (Figure 3C,D) Likewise, in nutrient-rich media and in minimal media (both solid and liquid), the deletion mutant produced fewer micro conidia compared to the wild-type strain. On the other hand, in overexpression mutants in both conditions, the number of recovered micro conidia was comparable with wild type (Figure S4C,D, Supplementary Materials). Hence, these results revealed that autophagy is important for mycelium growth and conidial formation in F. oxysporum. 

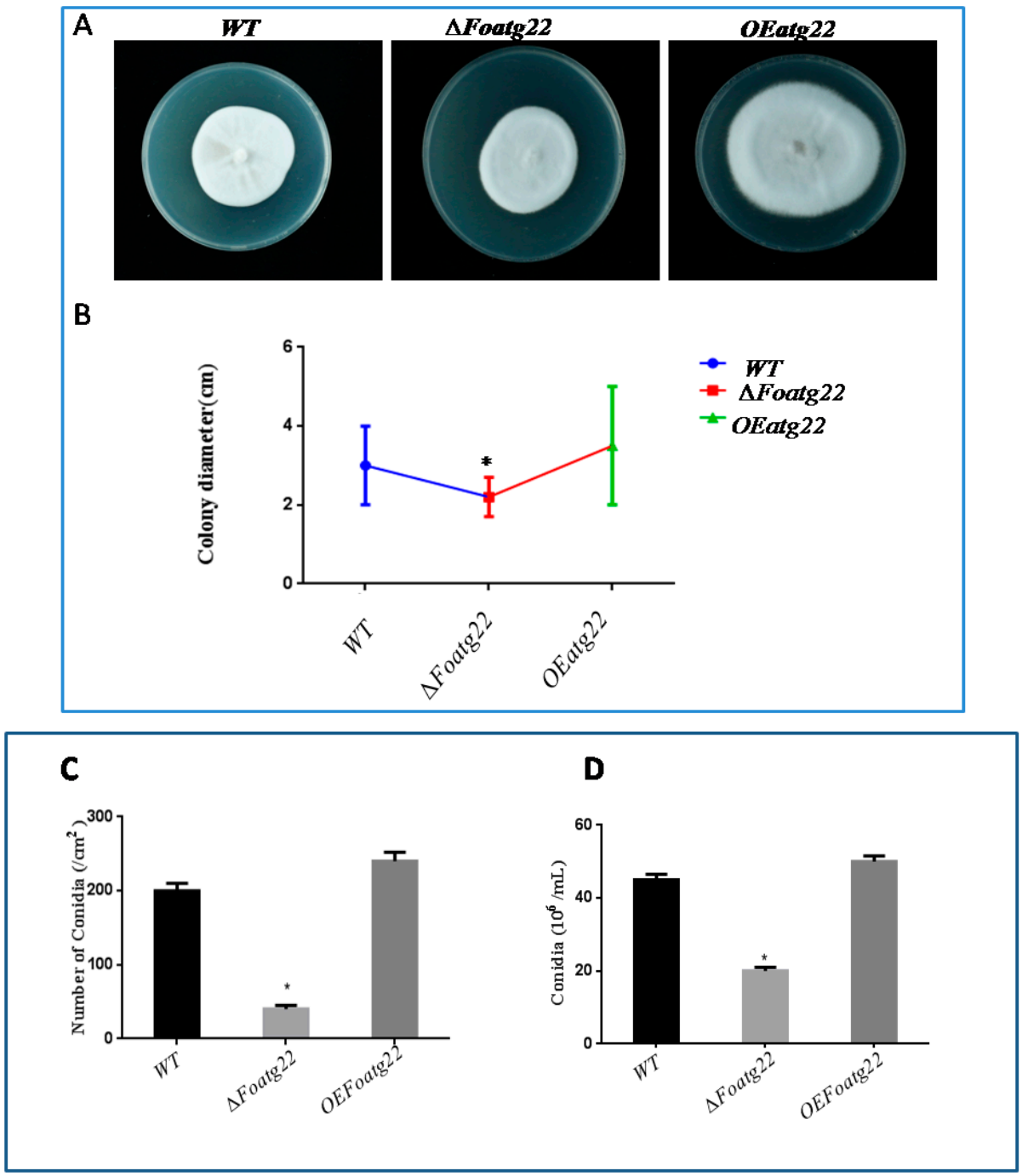

Figure 3. In $\Delta$ Foatg22mutants, conidial formation and hyphal formation was reduced. (A) Image of 14-day-old strains after inoculation. (B) Fresh micro conidia $\left(10^{3}\right)$ were inoculated on potato dextrose agar (PDA) plates and then incubated at $28^{\circ} \mathrm{C}$. Colony diameter was measured on a daily basis until two weeks. The graph represents the growth rate of strains. (C)Number of micro conidia recovered from PDA plates grown after 14 days, at $28^{\circ} \mathrm{C}$. Conidial formation was significantly reduced in $\Delta$ Foatg 22. (D) The number of recovered micro conidia from a two-day-old culture with shaking at $28{ }^{\circ} \mathrm{C}$. Conidial formation was reduced significantly in $\Delta$ Foatg22. Experiments were performed three times with similar results. Bars indicate standard error from replications. To perform statistical analysis, the Duncan post hoc test was used $(p<0.05)$.

\subsection{Expression of Vegetative Growth-Related Gene}

To investigate whether Foatg22 stimulates or inhibits the expression of genes for the production of conidia in F. oxysporum, the vegetative growth-related gene was evaluated by qRT-PCR. Firstly, the expression of hydrophobin-encoding FOXG_04522 was examined, which is an important factor for conidial formation in F. oxysporum. As compared with WT strains, the gene FOXG_04522 was not expressed in the deletion mutant, while the expression level of the overexpression mutant was not significantly different from wild type (Figure 4). 


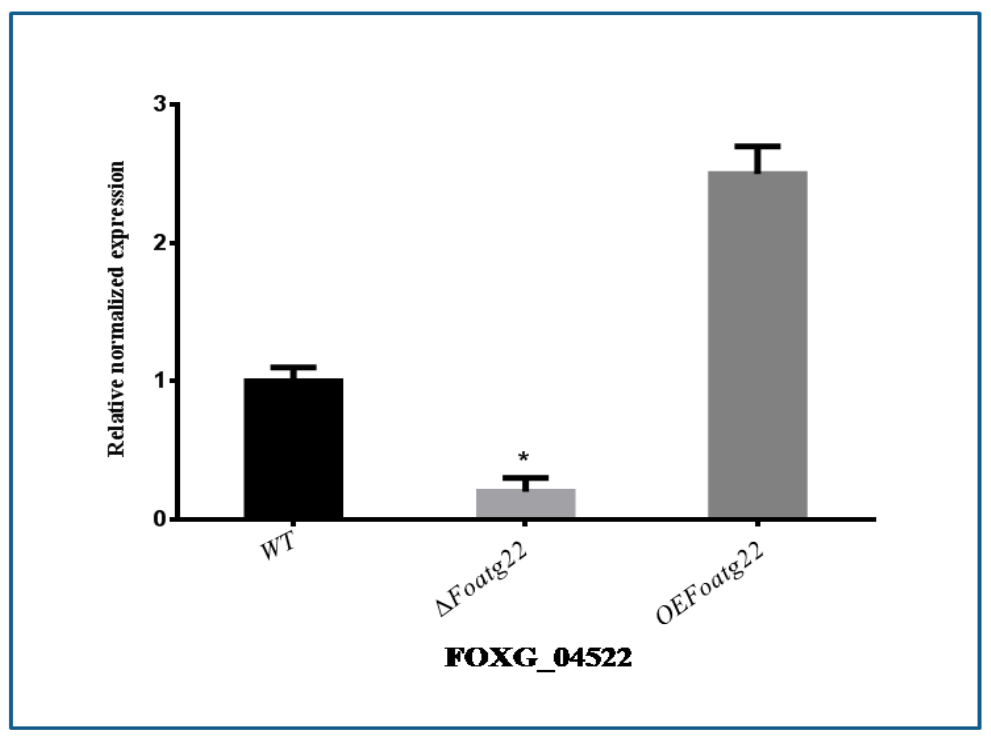

Figure 4. Verification of mutants by qRT-PCR. Quantitative real-time reverse-transcription polymerase chain reaction (qRT-PCR) analysis of vegetative growth-related gene in wild-type (WT), $\triangle$ Foatg22, and overexpression strains. Three biological replicates were used for this study. Statistical analysis was performed using the Duncan post hoc test $(p<0.05)$.

\subsection{Plant Infection Assay}

To investigate the role of the deletion mutant in the pathogenicity of F. oxysporum, potato (Solanum tuberosum) tubers and leaves were used for an infection assay. The potato tubers were inoculated with transgenic strains of $F$. oxysporum. The potato tuber inoculated by wild-type (WT) strains of F. oxysporum exhibited progressive symptoms of dry rot disease after seven days of inoculation. Notably, no detectable disease symptoms were observed on those potato tubers which were inoculated with the gene deletion mutant, and symptom development was slightly delayed compared to wild type (WT). The tubers which were inoculated with overexpression $(\mathrm{OE})$ mutants increased the progress of symptom development compared to wild type (WT) and the deletion mutant (Figure 5A,B). These findings suggest that autophagy-related gene Foatg 22 plays a role in the process of infection in F. oxysporum during early stages of the disease.

Furthermore, we also used detached potato leaves to test the pathogenicity of deletion mutant of $\Delta$ Foatg22. After seven days of inoculation, the deletion mutant $\Delta$ Foatg22 slightly reduced symptom development on potato leaves compared to wild-type and overexpression strains (Figure 5C). Taken together, these results showed that autophagy-related gene Foatg22 plays an important role in the pathogenicity of $F$. oxysporum.

Moreover, to determine the cause of failure of pathogenicity in the $\Delta$ Foatg22 mutant, the infection process was examined microscopically in detail after inoculation. Filamentous fungi start their disease cycle with the germination of conidia. Microscopic analysis showed that, after six days of inoculation, all strains produced conidia spores. The $\Delta$ Foatg 22 mutant exhibited a low concentration of conidia as compared to the wild type and the overexpression mutant (Figure $6 \mathrm{~A}, \mathrm{~B}$ ). Inability to produce conidia spores, however, can be an important component in the failure of $\Delta$ Foatg22 mutant infectivity. 


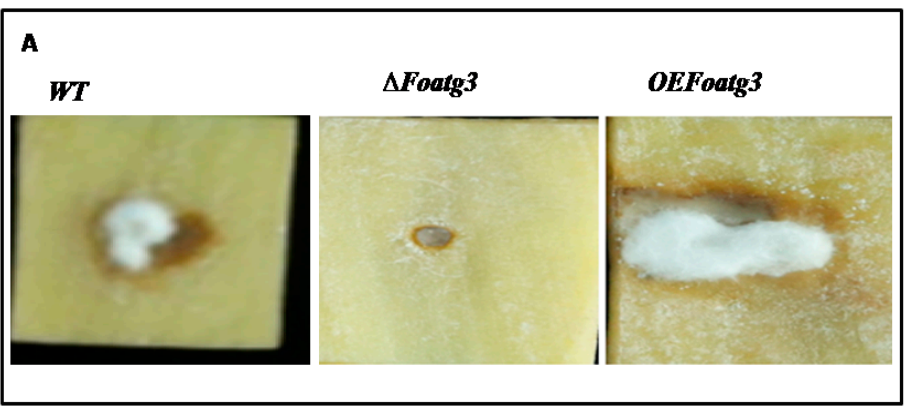

B

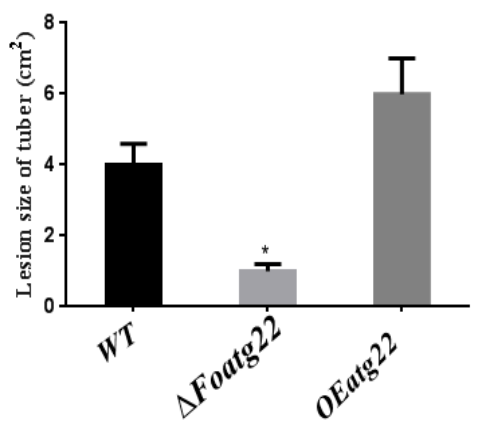

C

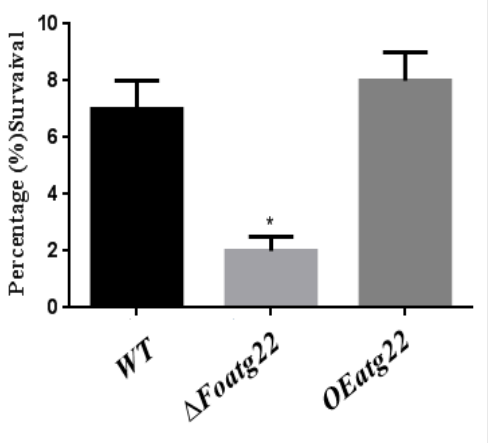

Figure 5. Foatg22 contributes to virulence on plant. (A) Disease progress in the tubers which were inoculated with wild type (WT), $\triangle$ Foatg22, and overexpression (OE) atg22. (B) Potato tubers were inoculated with a suspension of $5 \times 10^{6}$ freshly obtained micro conidia per $\mathrm{mL}$ of wild-type (WT), $\triangle$ Foatg22, and OE Foatg22 strains. Percentage of symptoms was recorded after seven days. (C) Potato leaves for each treatment were inoculated with a suspension of $5 \times 10^{6}$ freshly obtained micro conidia per $\mathrm{mL}$ of wild-type (WT), $\Delta$ Foatg22, and OEFoatg22 strains. Three replicates were used for all experiments with similar results (Duncan, $p<0.05$ ).

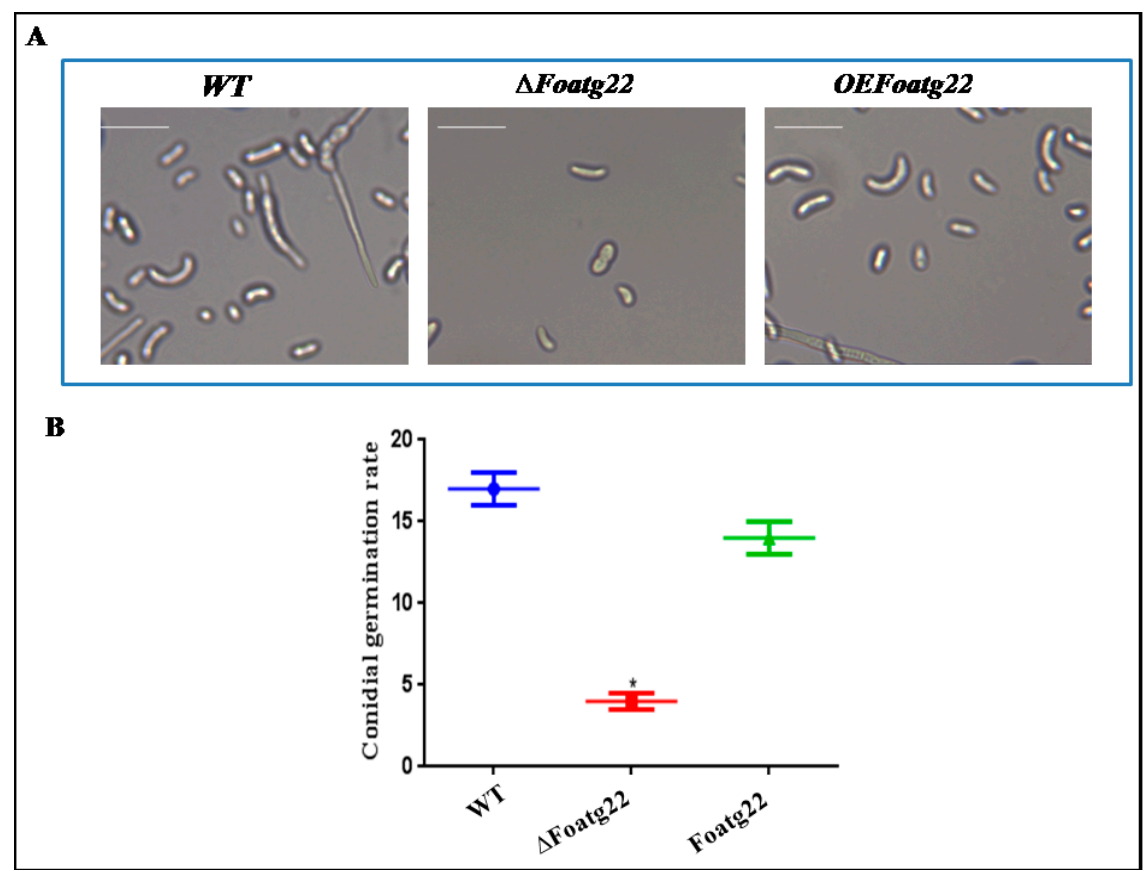

Figure 6. Hyphal germination was reduced in $\Delta$ Foatg22 mutants: (A) conidial germination; (B) conidial germination rate. Bars indicate the standard error from three independent replications (Duncan $<0.05$ ); Bars $=10 \mu \mathrm{m}$. 


\section{Discussion}

Previous studies in the filamentous fungi F. oxysporum showed that hyphae exhibit an acropetal vegetative growth. Subsequently, F. oxysporum can be considered as a mono nucleated compartmented mycelial organism [38]. This proposes the nearness of a system that decisively controls cell volume, compartmentalization, and cell cycle, subsequently keeping up the vegetative development and combination. In the last decade, a number of studies were carried out to explore the role of autophagy-related genes in filamentous fungi Colletotrichum orbiculare, Fusarium oxysporum, and Magnaporthe oryza, $[12,17,18]$. However, it is still unknown whether atg22 participates in autophagosome formation. Here, we found that the deletion mutant of atg22 suppressed autophagosome formation compared to wild type. On the other hand, in the overexpression mutant, formation of autophagosomes was enhanced compared to the deletion mutant and wild type. These findings revealed that $\operatorname{atg} 22$ may contain an important function to regulate autophagosome formation in F. oxysporum. Also, this finding shows a difference from previously reported functions of atg22 in yeast and F. oxysporum, which may be due to diverse functions in different species, and this can provide new insights for further studies [12,13]. The nonappearance of MDC-positive staining in $\Delta$ Foatg22 emphatically proposes that Foatg22 is a basic component for autophagy in F. oxysporum.

Endogenous recycling of the cytosol and organelles via autophagy is recommended to be vital for nutrient transport along hyphal filaments, as well as for the improvement of aerial hyphae which contain conidiophores $[39,40]$. In line with this thought, $\Delta$ Foatg22 strains displayed diminished aerial hyphae and conidiation, as reported in different filamentous fungi [33-37]. In virulent pathogens, successful virulence might rely upon the reusing of macromolecules to help cell movement under nutrient-restricting conditions. Filamentous fungi start their disease cycle with the germination of conidia. In M. oryzae, loss of MoATG8 resulted in autophagic conidial cell demise, prompting hindered appressorium arrangement, loss of pathogenicity, and decreased conidiation [16]. As shown in its capacity in M. oryzae, CoATG8 is moreover associated with conidiation, appressorium arrangement, and pathogenicity in C. orbiculare [17].

In a deletion mutant of aoatg8, conidial formation was delayed, which revealed that, in filamentous fungi, autophagy is required during early stages of conidial germination [36]. In agreement with previous studies, we found that the virulence of $\Delta$ Foatg22 strains on both potato [12] tubers and on detached leaves was significantly attenuated, producing fewer conidia spores compared to wild type and overexpression mutant; however, this can be an important component in the failure of the $\Delta$ Foatg22 mutant.

The genes $V d P K A C 1$ and $V d S N F 1$ are the known responsible factors for virulence in eggplant and tomato, while $V M K 1$ is also responsible for virulence on both tomato and tobacco, and VdNLP1 and $V d N L P 2$ are important factors in the virulence of cotton, Arabidopsis, and tobacco [10,11,25-27,41]. Autophagy plays an important role in the vegetative growth of F. oxysporum [11]. In the present study, the gene FOXG_04522, which is a well-known vegetative growth-related gene of F. oxysporum, was used. The deletion mutant of Foatg22 inhibited the expression level of pathogenicity-related genes during conidial formation. However, gene expression was consistent with phenotype. Finally, these findings verified the unreported assumption that atg 22 is essential for the formation of autophagosomes during the vegetative growth of F. oxysporum. Our findings strongly recommend that autophagy influences F. oxysporum to regulate vegetative growth. However, further studies are required to explore the exact role of atg22 during vegetative growth and the formation of autophagosomes.

Supplementary Materials: The following are available online at http://www.mdpi.com/2073-4425/10/5/365/s1; Figure S1: Construction of deletion and overexpressed mutants; Figure S2: Molecular identification of mutants; Figure S3: Representative hyphal growth and length of WT, $\Delta$ Foatg22, Overexpression of Foatg22.; Figure S4: Representative phenotype of WT, $\Delta$ Foatg22 and Overexpression of Foatg22.; Table S1: Primers used in present study.

Author Contributions: Data curation, Shumin Zhang; formal analysis, Xiumei Luo; investigation, A. Rehman Khalid, Khalid Mehmood, and HamayunShaheen; methodology, A. Rehman Khalid; project administration, A. Rehman Khalid; software, Maozhi Ren; visualization, Dan Qui; writing—original draft, A. Rehman Khalid; writing-review and editing, Maozhi Ren. 
Acknowledgments: This work was supported by grants from the National Key R\&D Program of China (2018YFD0200808 and 2016YFD0100306), the National Natural Science Foundation of China (No. 31672206 and 31801911), the Chongqing Frontier and Applied Basic Research (No. cstc2018jcyjAX0753 and cstc2015shmsztzx0121), and the Fundamental Research Funds for the Central Universities (No. 2018CDGFSM0021 and 106112017CDJQJ29883). National Key R\&D Program “Integrated Research and Demonstration of Potato Fertilizer and Pesticide Reduction Technology (2018YFD0200800)", Sub-project Name: Construction of Potato Weight Loss and Detoxification Demonstration Zone in Three Gorges Reservoir Area, Project No. 2018YFD020080804.

Conflicts of Interest: The authors declare no conflicts of interest.

\section{Abbreviations}

$\begin{array}{ll}\text { ATG } & \text { Autophagy-related } \\ \text { WT } & \text { Wild type } \\ \text { OE } & \text { Overexpression } \\ \Delta & \text { Deletion mutant } \\ \text { MDC } & \text { Monodansylcadaverine } \\ \text { DIC } & \text { Differential interference contrast }\end{array}$

\section{References}

1. El-Kassas, H.Y.; Khairy, H.M. A trial for biological control of a pathogenic fungus (Fusarium solani) by some marine microorganisms. Am. Eurasian. J. Agric. Environ. Sci. 2009, 3, 434-440.

2. Wharton, P.K.W. Fusarium Dry Rot. Available online: http://www.Potatodiseases.Org/contact.html (accessed on 23 May 2007).

3. Wharton, P.S.; Kirk, W.W.; Berry, D.; Tumbalam, P. Seed treatment application-timing options for control of Fusarium decay and sprout rot of cut seedpieces. Am. J. Potato Res. 2007, 3, 237-244. [CrossRef]

4. Chełkowski, J. Toxinogenicity of Fusarium Species Causing Dry rot of Potato Tubers - Fusarium-Chapter 25; Elsevier B.V.: New York, NY, USA, 1989; pp. 435-440.

5. Slininger, P.J.; Burkhead, K.D.; Schisler, D.A. Antifungal and sprout regulatory bioactivities of phenylacetic acid, indole-3-acetic acid, and tyrosol isolated from the potato dry rot suppressive bacterium Enterobacter cloacae S11 : T : 07. J. Ind. Microbiol. Biotechnol. 2004, 11, 517-524. [CrossRef] [PubMed]

6. Chełkowski, J. Chapter 25 - Toxinogenicity of fusarium species causing dry rot of potato tubers. In Fusarium; Chełkowski, J., Ed.; Elsevier: Amsterdam, The Netherlands, 1989; Volume 2, pp. 435-440.

7. Secor, G.A.; Gudmestad, N.C. Managing fungal diseases of potato. Can. J. Plant Pathol. 1999, 3, $213-221$. [CrossRef]

8. Shattock, R. Compendium of Potato Diseases, Second Edition. W.R. Stevenson. Plant Pathol. 2002, 4. [CrossRef]

9. Secor, G.; Salas, B. Fusarium dry rot and Fusarium wilt. Compend. Potato Dis. 2001, 23-25.

10. Santhanam, P.; van Esse, H.P.; Albert, I.; Faino, L.; Nurnberger, T.; Thomma, B.P.H.J. Evidence for functional diversification within a fungal nep1-like protein family. Mol. Plant Microbe Interact. 2013, 3, 278-286. [CrossRef]

11. Corral-Ramos, C.; Roca, M.G.; Di Pietro, A.; Roncero, M.I.G.; Ruiz-Roldan, C. Autophagy contributes to regulation of nuclear dynamics during vegetative growth and hyphal fusion in Fusarium oxysporum. Autophagy 2015, 1, 131-144. [CrossRef] [PubMed]

12. Sun, L.L.; Li, M.; Suo, F.; Liu, X.M.; Shen, E.Z.; Yang, B.; Dong, M.Q.; He, W.Z.; Du, L.L. Global analysis of fission yeast mating genes reveals new autophagy factors. PLoS Genet. 2013, 8, 244-258.

13. Chardwiriyapreecha, S.; Mukaiyama, H.; Sekito, T.; Iwaki, T.; Takegawa, K.; Kakinuma, Y. Avt5p is required for vacuolar uptake of amino acids in the fission yeast Schizosaccharomyces pombe. FEBS Lett. 2010, 11, 2339-2345. [CrossRef] [PubMed]

14. Suriapranata, I.; Epple, U.D.; Bernreuther, D.; Bredschneider, M.; Sovarasteanu, K.; Thumm, M. The breakdown of autophagic vesicles inside the vacuole depends on Aut4p. J. Cell Sci. 2000, 22, 4025-4033.

15. Veneault-Fourrey, C.; Barooah, M.; Egan, M.; Wakley, G.; Talbot, N.J. Autophagic fungal cell death is necessary for infection by the rice blast fungus. Science 2006, 5773, 580-583. [CrossRef]

16. Asakura, M.; Ninomiya, S.; Oku, M.; Okuno, T.; Sakai, Y.; Takano, Y. Atg26-mediated pexophagy is required for host invasion by the plant pathogenic fungus Colletotrichum orbiculare. Autophagy 2009, 6.

17. Dong, B.; Liu, X.H.; Lu, J.P.; Zhang, F.S.; Gao, H.M.; Wang, H.K.; Lin, F.C. MgAtg9 trafficking in Magnaportheoryzae. Autophagy 2009, 7, 946-953. [CrossRef] 
18. Lu, J.P.; Liu, X.H.; Feng, X.X.; Min, H.; Lin, F.C. An autophagy gene, $M g A T G 5$, is required for cell differentiation and pathogenesis in Magnaportheoryzae. Curr. Genet. 2009, 4, 461-473. [CrossRef]

19. Nadal, M.; Gold, S.E. The autophagy genes atg8 and atg1 affect morphogenesis and pathogenicity in Ustilago maydis. Mol. Plant Pathol. 2010, 4, 463-478. [CrossRef] [PubMed]

20. Lv, W.; Wang, C.; Yang, N.; Que, Y.; Talbot, N.J.; Wang, Z. Genome-wide functional analysis reveals that autophagy is necessary for growth, sporulation, deoxynivalenol production and virulence in Fusarium graminearum. Sci. Rep. 2017, 1, 110-125. [CrossRef]

21. Liu, X.H.; Lu, J.P.; Zhang, L.; Dong, B.; Min, H.; Lin, F.C. Involvement of a magnaporthegrisea serine/threonine kinase gene, mgatg1, in appressorium turgor and pathogenesis. Eukaryot. Cell 2007, 6, 997-1005. [CrossRef]

22. Liu, T.B.; Liu, X.H.; Lu, J.P.; Zhang, L.; Min, H.; Lin, F.C. The cysteine protease MoAtg4 interacts with MoAtg8 and is required for differentiation and pathogenesis in Magnaportheoryzae. Autophagy 2010, 1, 74-85. [CrossRef]

23. Kershaw, M.J.; Talbot, N.J. Genome-wide functional analysis reveals that infection-associated fungal autophagy is necessary for rice blast disease. Proc. Natl. Acad. Sci. USA 2009, 37, 15967-15972. [CrossRef]

24. Rauyaree, P.; Ospina-Giraldo, M.D.; Kang, S.; Bhat, R.G.; Subbarao, K.V.; Grant, S.J.; Dobinson, K.F. Mutations in $V M K 1$, a mitogen-activated protein kinase gene, affect microsclerotia formation and pathogenicity in Verticillium dahliae. Curr. Genet. 2005, 2, 109-116. [CrossRef]

25. Tzima, A.; Paplomatas, E.J.; Rauyaree, P.; Kang, S. Roles of the catalytic subunit of cAMP-dependent protein kinase A in virulence and development of the soilborne plant pathogen Verticillium dahliae. Fungal Genet. Biol. 2010, 5, 406-415. [CrossRef]

26. Tzima, A.K.; Paplomatas, E.J.; Rauyaree, P.; Ospina-Giraldo, M.D.; Kang, S. VdSNF1, the Sucrose nonfermenting protein kinase gene of Verticillium dahliae, is required for virulence and expression of genes involved in cell-wall degradation. Mol. Plant Microbe Interact. 2011, 1, 129-142. [CrossRef] [PubMed]

27. Tzima, A.K.; Paplomatas, E.J.; Tsitsigiannis, D.I.; Kang, S. The G protein $\beta$ subunit controls virulence and multiple growth- and development-related traits in Verticillium dahliae. Fungal Genet. Biol. 2012, 4, 271-283. [CrossRef] [PubMed]

28. Szewczyk, E.; Nayak, T.; Oakley, C.E.; Edgerton, H.; Xiong, Y.; Taheri-Talesh, N.; Osmani, S.A.; Oakley, B.R. Fusion PCR and gene targeting in Aspergillus nidulans. Nat. Protoc. 2006, 6, 3111-3120. [CrossRef]

29. Maruthachalam, K.; Klosterman, S.J.; Kang, S.; Hayes, R.J.; Subbarao, K.V. Identification of pathogenicityrelated genes in the vascular wilt fungus Verticillium dahliae by Agrobacterium tumefaciens-mediated t-dna insertional mutagenesis. Mol. Biotechnol. 2011, 3, 209-221. [CrossRef]

30. Di Pietro, A.; Roncero, M.I.G. Cloning, expression, and role in pathogenicity of pg1 encoding the major extracellular endopolygalacturonase of the vascular wilt pathogen Fusarium oxysporum. Mol. Plant Microbe Interact. 1998, 2, 91-98. [CrossRef]

31. Biederbick, A.; Kern, H.F.; Elsasser, H.P. Monodansylcadaverine (Mdc) is a specific in-vivo marker for autophagic vacuoles. Eur. J. Cell Biol. 1995, 1, 3-14.

32. Bartoszewska, M.; Kiel, J.A.K.W. The role of macroautophagy in development of filamentous fungi. Antioxid. Redox Sign. 2011, 11, 2271-2287. [CrossRef]

33. Josefsen, L.; Droce, A.; Sondergaard, T.E.; Sorensen, J.L.; Bormann, J.; Schafer, W.; Giese, H.; Olsson, S. Autophagy provides nutrients for nonassimilating fungal structures and is necessary for plant colonization but not for infection in the necrotrophic plant pathogen Fusarium graminearum. Autophagy 2012, 3, 326-337. [CrossRef] [PubMed]

34. Kikuma, T.; Ohneda, M.; Arioka, M.; Kitamoto, K. Functional analysis of the ATG8 homologue Aoatg8 and role of autophagy in differentiation and germination in Aspergillus oryzae. Eukaryot. Cell 2006, 8, 1328-1336. [CrossRef] [PubMed]

35. Duan, Z.B.; Chen, Y.X.; Huang, W.; Shang, Y.F.; Chen, P.L.; Wang, C.S. Linkage of autophagy to fungal development, lipid storage and virulence in Metarhiziumrobertsii. Autophagy 2013, 4, 538-549. [CrossRef]

36. Nitsche, B.M.; Burggraaf-van Welzen, A.M.; Lamers, G.; Meyer, V.; Ram, A.F.J. Autophagy promotes survival in aging submerged cultures of the filamentous fungus Aspergillus niger. Appl. Microbiol. Biotechnol. 2013, 18, 8205-8218. [CrossRef] [PubMed]

37. Ruiz-Roldan, M.C.; Kohli, M.; Roncero, M.I.G.; Philippsen, P.; Di Pietro, A.; Espeso, E.A. Nuclear Dynamics during Germination, Conidiation, and Hyphal Fusion of Fusarium oxysporum. Eukaryot. Cell 2010, 8, 1216-1224. [CrossRef] [PubMed] 
38. Richie, D.L.; Fuller, K.K.; Fortwendel, J.; Miley, M.D.; McCarthy, J.W.; Feldmesser, M.; Rhodes, J.C.; Askew, D.S. Unexpected link between metal ion deficiency and autophagy in Aspergillus fumigatus. Eukaryot. Cell 2007, 12, 2437-2447. [CrossRef]

39. Shoji, J.Y.; Arioka, M.; Kitamoto, K. Possible involvement of pleiomorphic vacuolar networks in nutrient recycling in filamentous fungi. Autophagy 2006, 3, 226-227. [CrossRef]

40. Luo, X.M.; Mao, H.Q.; Wei, Y.M.; Cai, J.; Xie, C.J.; Sui, A.P.; Yang, X.Y.; Dong, J.Y. The fungal-specific transcription factor Vdpf influences conidia production, melanized microsclerotia formation and pathogenicity in Verticillium dahliae. Mol. Plant Pathol. 2016, 9, 1364-1381.

41. Zhou, L.; Zhao, J.; Guo, W.Z.; Zhang, T.Z. Functional Analysis of Autophagy Genes via AgrobacteriumMediated Transformation in the Vascular Wilt Fungus Verticillium dahliae. J. Genet. Genom. 2013, 8, 421-431. [CrossRef] [PubMed]

(C) 2019 by the authors. Licensee MDPI, Basel, Switzerland. This article is an open access article distributed under the terms and conditions of the Creative Commons Attribution (CC BY) license (http://creativecommons.org/licenses/by/4.0/). 